\title{
The influence of nonnormality from primary studies on the standardized mean difference in meta-analysis
}

\author{
Rong Wei Sun ${ }^{1} \cdot$ Shu Fai Cheung ${ }^{1}$ \\ Published online: 2 January 2020 \\ (C) The Psychonomic Society, Inc. 2020
}

\begin{abstract}
In this study we investigated the influence of data nonnormality in the primary studies on meta-analysis of the standardized mean difference (SMD) for a two-independent-group design. The bias, mean squared error, and confidence interval coverage probability of the mean effect sizes under different types of population distributions were compared. Also, the performance of the $Q$ test was examined. The results showed that oppositely skewed distributions (i.e., distributions skewed in different directions) showed poor performance for point and interval estimates of mean effect sizes in meta-analysis, especially when the tails were pointing toward each other. The previously found adverse impacts due to nonnormality in primary studies do not disappear when primary studies with nonnormal data are meta-analyzed, even when the average sample size and number of studies are large. The results also showed that, when the tails were pointing toward each other, the Type I error rates of the $Q$ test were inflated. We suggest that the impact of violating the assumption of normality should not be ignored in meta-analysis.
\end{abstract}

Keywords Meta-analysis $\cdot$ Nonnormality $\cdot$ Standardized mean difference $\cdot Q$ tests

Meta-analysis is used to summarize and integrate quantitative data extracted from the available studies on a specific topic. The cases in meta-analyses are studies instead of individuals, and these studies are called primary studies in meta-analysis. When the topic is the mean difference between two independent groups, such as a control group and a treatment group, one of the most commonly applied effect size measures is the standardized mean difference (SMD), which standardizes the mean difference between two independent groups using a relevant standard deviation (e.g., the standard deviation of the control group or the common standard deviation when the two groups are assumed to have the same population standard deviation). Like the $z$ score (i.e., the deviation from the mean, in standard deviations), this dimensionless measure is useful when the primary studies used different units. The SMD is easy to interpret and estimate when the variable of concern is normally distributed in the population in both groups, such that the only difference between the two groups on this variable is the population mean if the two groups have the same population standard deviation. However, it is not unusual for

Rong Wei Sun

irissun_s@hotmail.com

1 Department of Psychology, University of Macau, Macao, SAR, China this assumption of normality to be violated. We will discuss two ways in which this assumption is violated - the population distributions of the two groups are the same but nonnormal, or only one of the population distributions is normal (e.g., one is a normal distribution and another is an exponential distribution) - and how these cases may affect the meta-analysis of SMD.

\section{Nonnormality and heteroscedasticity in primary studies}

SMD (denoted by $\delta$ below), which standardizes the mean difference between two groups, is not robust to nonnormality and heteroscedasticity (i.e., unequal population variances in the two groups). With the assumption of normality (Hedges, 1981, p. 108; Hedges \& Olkin, 1985, p. 76), the SMD is defined by

$\delta=\frac{\mu_{1}-\mu_{2}}{\sigma}$,

where $\mu_{1}$ and $\mu_{2}$ are the population means of the two populations being compared, and $\sigma$, the population standard deviation, is called the standardizer.

On the one hand, one problem of SMD mentioned by Algina, Keselman, and Penfield (2005) is that the population 
mean and standard deviation are least-square parameters. As was shown by Staudte and Sheather (1990), least-square parameters are not robust and are sensitive to nonnormality in the population distribution. On the other hand, if the assumptions of the normality and homogeneity of the population variances are both violated, interpretation of the SMD based on the distribution overlapping is hazardous. As Bonett (2008) noted, if the population distributions in both groups are normal, an SMD of 0.5 can easily be visualized as two standard normal curves separated by 0.5 standard deviations. However, the SMD cannot be easily interpreted in this way when the distributions are not normal. In addition, with the violation of equal variances, "a standardized measure of treatment effects comparable to [SMD] would seem not to exist" (Wilcox, 1987, p. 47).

\section{Estimators of SMD}

The most popular estimator of SMD is Hedges's $g$. It is a consistent and asymptotically efficient estimator if the population distributions are normal (Hedges \& Olkin, 1985). The definition of Hedges's $g$, which is the Cohen's $d$ multiplied by a correction factor, is given by Hedges and Olkin (1985, Eq. 10, p. 81):

$g=\frac{\overline{X_{1}}-\overline{X_{2}}}{s_{p}} \times\left\{1-\frac{3}{4\left(n_{1}+n_{2}\right)-9}\right\}$,

where $\overline{X_{1}}$ and $\overline{X_{2}}$ are the sample means of Group 1 and Group 2 , respectively, $n_{1}$ and $n_{2}$ are the sample sizes of Group 1 and Group 2, respectively, and $s_{p}$ is the pooled sample standard deviation of Group 1 and Group 2-that is,

$s_{p}=\sqrt{\frac{\left(n_{1}-1\right) s_{1}^{2}+\left(n_{2}-1\right) s_{2}^{2}}{N-2}}$,

where $N=n_{1}+n_{2}$, and $s_{1}$ and $s_{2}$ are the sample standard deviations of Group 1 and Group 2, respectively. The calculation of $s_{p}$ assumes the homogeneity of variances.

The estimator of the sampling variance of Hedges's $g, \partial_{g}^{2}$, is given by Hedges and Olkin (1985, Eq. 15, p. 86):

$\partial_{g}^{2}=\frac{N}{n_{1} n_{2}}+\frac{g^{2}}{2 N}$.

\section{Nonnormality and heteroscedasticity on SMD estimation}

One may think that, due to the central limit theorem (CLT), Hedges's $g$ is still unbiased under nonnormality. CLT states that, regardless of the shape of the distribution, the sums or means of independent and identically distributed data, with sufficiently large samples, will be normally distributed (Micceri, 1989). However, the CLT cannot be generalized to Hedges's $g$.Note that Cohen's $d$ can be computed from the value of the $t$ test ( $t$ multiplied by a constant that depends on sample sizes only; Rosenthal \& Rosnow, 1991), and Hedges's $g$ can be computed from Cohen's $d$ (as is shown in Eq. 2 above, Cohen's $d$ multiplied by a constant that depends on the sample size only). In this way, the distribution of Hedges's $g$ approaches normal as the distribution of the sample $t$ distribution approaches normal, which happens when the degree of freedom increases (i.e., the total sample size increases). However, simulation studies (e.g., Cribbie, Fiksenbaum, Keselman, \& Wilcox, 2012; Sawilowsky \& Blair, 1992) have shown that the $t$ test is not robust when the population distribution is extremely skewed. Therefore, the adverse impact of nonnormality of the raw data on a $t$ test could also affect the estimation of Hedges's $g$.

Another problem is caused by the variance of the sampling distribution of $s^{2}$. In Hedges's $g, s^{2}$ is used as the standardizer. As was shown by Games and Hedges (1987), the variance of the sampling distribution of $s^{2}$ is $V\left(s^{2}\right)=\left(\sigma^{2}\right)^{2}\left(\frac{2}{n-1}+\gamma_{2} / n\right)$, where $\gamma_{2}$ is excess kurtosis and $\sigma^{2}$ is population variance. If the kurtosis is large, then $V\left(s^{2}\right)$ is large, which will yield an inflated Type I error rates of parametric tests (e.g., $t$ test). It is well known kurtosis has an adverse effect on the estimation of SMD (e.g., Algina et al., 2005; Games, Keselman, \& Clinch, 1979).

In psychological and educational areas, the problem of nonnormality is more common than usually assumed. For example, Micceri (1989) reviewed 440 large-sample psychological and educational measures and found that only a few datasets had approximately normal distributions. One recent study by Blanca, Arnau, López-Montiel, Bono, and Bendayan (2013) analyzed measures of cognitive ability and other psychological variables (693 distributions with sample sizes ranging from 10 to 30 ) and also found that normality is rare and nonnormality appears to be the norm. Therefore, for metaanalysts, when using SMD as the effect size measure, the assumption of normally distributed raw data, and consequently the assumption of normally distributed sample SMD (e.g., Hedges's $g$ ), may not be tenable for most, if not all, primary studies. That is, nonnormality in primary studies probably can influence the validity of the meta-analytic results.

\section{Nonhomomerity on SMD estimation}

Nonhomomerity is a special case of nonnormality, in which the population distributional shapes are not the same for the two independent groups (Grissom \& Kim, 2001). Studies have shown that nonhomomerity, such as one distribution is 
normal and another is skewed, is not rare in educational and psychological studies. Examples are sex differences in intellectual abilities (Feingold, 1992) and comparisons between schizophrenic patients and control group on the nonverbal language (Leentjens, Wielaert, van Harskamp, \& Wilmink, 1998; see Bendayan, Arnau, Blanca, \& Bono, 2013, for other examples). The influence of nonhomomerity on SMD could be inferred from previous simulations on the $t$ test (e.g., Cribbie et al., 2012; Delaney \& Vargha, 2000; Tiku, 1964). For example, Tiku (1964) showed the large effects of oppositely skewed populations (one population positively skewed and the other negatively skewed) on the $t$ test. Cribbie et al. (2012) found similar results for Welch's $F$ test that the Type I error rates under skewed distributions increased substantially, especially in oppositely skewed distributions. They also found that the power rates of Welch's $F$ test for skewed distributions are substantially lower than those for normal distributions. Finally, the simulation by Delaney and Vargha (2000) showed that, for skewed distributions, the Type I error rates of $t$ tests were much higher than the nominal level, especially for oppositely skewed distributions. Thus, given the relation between Hedges's $g$ and the $t$ statistic shown above (Rosenthal \& Rosnow, 1991), there is no doubt that nonhomomerity could influence the estimation of SMD in individual studies.

\section{Nonnormality and nonhomomerity in meta-analysis}

On the one hand, Hedges's $g$ and its sampling variance are derived from the properties of noncentral $t$ distribution (Hedges, 1981), which assumes that the population distributions of the two groups are normal. It could be speculated that nonnormality could influence not only the estimation of the population SMD by Hedges's $g$, but also its sampling variance.

On the other hand, the sampling variance of Hedges's $g$ depends on the sample Hedges's $g$ (shown in Eq. 4). In this way, the sampling variance of Hedges's $g$ is estimated, not fixed and known (Veroniki et al., 2018). To calculate overall effect size in a meta-analysis (see Eq. 6 below), it is hard to know whether sampling variances as the weights and the probable bias in Hedges's $g$ together could finally lead to a more biased overall effect size or balanced out to have a less biased overall effect size in meta-analysis. Also, the sampling variance of the Hedges's $g$ of each study is used to calculate the sampling variance of the overall effect size. It has been shown in many simulation studies of primary studies that the confidence interval coverage probability of estimates of SMD is lower than the nominal level when the assumptions of normality and homoscedasticity are violated (e.g., Algina, Keselman, \& Penfield, 2005, 2006; Hess \& Kromrey, 2004;
Kelley, 2005). The narrower confidence interval coverage means that the sampling variance of Hedges's $g$ is underestimated. The underestimated sampling variances from the individual studies will lead to overestimated weights, and summing up those inversed weights finally leads to underestimated the sampling variance in a meta-analysis (see Eqs. 9 and 11 below). Thus, both the overall effect size and its sampling variance in a meta-analysis might be influenced by nonnormality and heteroscedasticity in the primary studies.

Previous simulations have mainly focused on the influence of nonnormality in primary studies (e.g., Algina, et al., 2005; Bonett, 2008), and limited attention has been paid to assessing how nonnormality and nonhomomerity in the primary studies can affect meta-analytic results. We summarize the relevant simulations below.

Johnson and Huedo-Medina (2013) generated nonnormal data in primary studies to examine the estimation of SMD in meta-analysis. They showed that the bias of SMD's estimators (including Hedges's $g$ ) slightly increased and the efficiency decreased with increasing skewness and kurtosis in the primary studies (skewness/kurtosis $=0.5 / 0,0.75 / 0$, and 1.75/3.75), and the bias greatly increased with increasing variance ratios. However, they did not study nonhomomerity. Also, SánchezMeca, Marín-Martínez, and Chacón-Moscoso (2003) compared several effect size measures involving two groups (including Hedges's $g$ and odds ratio, the latter not studied in the present article) in meta-analysis. Their simulation results supported the robustness of Hedges's $g$ (denoted as $d$ in their study), which was hardly influenced by the degree of skewness (skewness/kurtosis $=0.5 / 0,0.75 / 0$, and 1.75/3.75). However, as in Johnson and Huedo-Medina's study, the distributions for the two groups were identical across conditions in their simulation.

Besides the estimation of effect size in meta-analysis, the influence of nonnormality on the $Q$ test, which is used to test the homogeneity of effect sizes across studies, was also examined by various studies (e.g., Harwell; Huedo-Medina, Jo, and Botella). Harwell (1997) found that skewed distributions in primary studies had little effect on the Type I error rate and power of the $Q$ test. However, a small study size coupled with large sample sizes tends to produce conservative Type I error rates and low statistical power for the $Q$ test (Harwell, 1997). A similar result was found in the simulation by HuedoMedina et al., (2006), showing that nonnormality alone did not affect the performance of the $Q$ test. Besides, their study examined the conditions with the violation of homoscedasticity, and their result showed dramatic increase in Type I error rate and decrease in power under these conditions. These results suggest that heteroscedasticity can influence the performance of a homogeneity test, but nonnormality has little effect on this test.

To date, no simulation study has examined nonhomomerity in meta-analyzing SMD estimates. Many questions remain 
unanswered about the influence of nonhomomerity, such as how oppositely skewed distributions in primary study may affect the performance of homogeneity test. To fill the research gap, in this article we investigate how nonnormality and nonhomomerity, especially oppositely skewed situations, influence the meta-analytic results using SMD as the effect size measure. Even though there are many different kinds of nonnormal data, we have included only two types of skewed distributions, the exponential distributions and the reversed exponential distributions, because they are more likely to have an impact on the meta-analytic results and they are also commonly found. Exponential distributions ${ }^{1}$ are widespread in the health and social sciences (Bono, Blanca, Arnau, \& GómezBenito, 2017). The exponential distribution is positively skewed with a heavy right tail. It has been used to model a wide range of variables, such as response times, physical and verbal violence in couples, the cost of health care among patients with depression or anxiety, and incremental differentiation (see more examples in Bono et al., 2017, and Joo, Aguinis, \& Bradley, 2017). More importantly, the exponential distribution and the reversed exponential distribution could be applied to model floor and ceiling effects. For example, for response time to a difficult task, many participants may fail to finish the task and have response time equal to the time allowed, while a few participants may finish the task and hence have low response times, resulting in a negatively skewed distribution. This ceiling effects could be modeled by the reversed exponential distribution. Exponential distributions are also commonly examined in Monte Carlo simulations (e.g., Harwell, Rubinstein, Hayes, \& Olds, 1992; Keselman, Algina, \& Fradette, 2005). In this simulation, using both the exponential and reversed exponential distributions allowed us to examine cases with oppositely skewed distributions.

\section{Method}

\section{Simulation design and data generation}

The meta-analytic data were simulated with the following factors, summarized in Table 1.

First, the mean population standardized mean difference, $\delta$, was manipulated with values of $0,0.2,0.5,0.8,1.1$, and 1.4. According to Cohen's (1988) rule of thumb for $d$, the levels of $0.2,0.5$, and 0.8 are considered as small, medium, and large effect sizes, respectively. Zero effect (0) was included in order to assess the Type I error rate, and two large effect sizes (1.1

\footnotetext{
${ }^{1}$ The exponential distribution we defined here is the standard exponential distribution with mean and standard deviation shifted and rescaled. It is positively skewed with a long right tail. It is also called a negative exponential distribution. The reversed exponential distribution is also called a positive exponential distribution, which is negatively skewed with a long left tail.
}

and 1.4) were included in order to evaluate more extreme conditions. In a recent review of 54 meta-analyses of clinical psychological treatment by Rubio-Aparicio, Marín-Martínez, Sánchez-Meca, and López-López (2018), the minimum estimated mean SMD in the reviewed meta-analytic studies is .068 , and the maximum is 1.075 . The range we examined covers and extends beyond this range.

Second, the $S D$ ratio, which is the ratio of the standard deviations of the two groups compared, was created with values of $1,2,4,0.5$, and 0.25 . A number of previous simulations (e.g., Huedo-Medina et al., 2006; Johnson \& HuedoMedina, 2013; Ruscio \& Mullen, 2012; Wilcox, 1987) have studied three levels of $S D$ ratio-namely, 1, 2, and 4-and have assumed that due to the experimental manipulation, the variance should be higher for the experimental group than for the control group. However, this is not always the case in practice. For example, Moè and Pazzaglia (2006) designed an experiment to detect the effect of gender beliefs in mental rotation. In one condition, the female participants had a mean of 9.69 with a standard deviation equal to 5.15 , whereas the male participants had a mean of 12.13 with a standard deviation of 2.70. The group with the higher mean did not have a higher standard deviation. Hence, we included 0.5 and 0.25 for unequal-variance situations. When the value of the $S D$ ratio is set to 1 , the assumption of homogeneity of variances is met. Conditions with $S D$ ratios not equal to 1 represent situations that violate the assumption of homogeneity.

Third, the average sample size across studies $(\bar{N})$ was varied, with values of $30,50,80$, and 100 . We specified four vectors for the total sample size within a study $N_{i}\left(N_{i}=n_{1 i}+\right.$ $n_{2 i}$ and $\left.n_{1 i}=n_{2 i}\right)$, which are $[12,16,18,20,84],[32,36,38$, $40,104]$, [62, 66, 68, 70, 134], and [82, 86, 88, 90, 154], with means of the vector elements equal to $30,50,80$, and 100 , respectively. The sample sizes were selected on the basis of a review of 30 meta-analyses by Sánchez-Meca and MarínMartínez (1998), in which the Pearson skewness index of sample sizes was equal to 1.464 (Marín-Martínez \& Sánchez-Meca, 2010). ${ }^{2}$ In each condition, one of the vectors of the desired average sample size was replicated $1,2,4,8$, or 20 times, depending on the desired number of studies. For instance, when the study size was equal to 10 and average sample size $(\bar{N})$ was equal to 50 , the corresponding vector $[32,36,38,40,104]$ was replicated twice. In other words, the total sample sizes within the study were $N_{i}=[32,36,38,40$, $104,32,36,38,40,104]$, and the sample sizes for the two groups for the 10 studies were $n_{1 i}=n_{2 i}=[16,18,19,20,52$, $16,18,19,20,52]$. This range of study sizes was found commonly in published meta-analyses. For instance, in the metameta-analysis by Cafri, Kromrey, and Brannick (2010), they

\footnotetext{
${ }^{2}$ In the article, Sánchez-Meca and Marín-Martínez (1998) also reported the mean sample size per study; the mean sample size was 79 , the median was 56 , and the standard deviation was 65 .
} 
Table 1 Summary of the parameters applied in the simulation

\begin{tabular}{|c|c|c|}
\hline IVs & Values & Descriptions \\
\hline $\begin{array}{l}\text { Effect sizes }(\delta, \text { six } \\
\quad \text { levels })\end{array}$ & $0,0.20,0.50,0.80,1.1$, and 1.4 & $\begin{array}{l}\text { The mean of Group } 1 \text { is fixed to } 0 \text {, and the standard deviation is } \\
\text { fixed to } 1 \text {; the mean of Group } 2 \text { is equal to } \\
\delta \sqrt{\left(S R^{2}+1\right) / 2} \text {, and the standard deviation is fixed to the } \\
S D \text { ratio. }\end{array}$ \\
\hline $\begin{array}{l}S D \text { ratio (five } \\
\text { levels) }\end{array}$ & $1,2,4, .5, .25$ & $\begin{array}{l}S D \text { ratio is the ratio of the standard deviations between two } \\
\text { groups. }\end{array}$ \\
\hline $\begin{array}{l}\text { Mean sample sizes } \\
(\bar{N}, \text { four levels })\end{array}$ & $30,50,80$, and 100 & $\begin{array}{l}\text { The sample sizes }\left(n_{1}=n_{2} \text { and } N=n_{1}+n_{2}\right) \text { are equal for the } \\
\text { two groups in the individual studies. }\end{array}$ \\
\hline $\begin{array}{l}\text { Study sizes ( } k \text {, five } \\
\text { levels) }\end{array}$ & $5,10,20,40$, and 100 & The number of studies included in one meta-analysis. \\
\hline $\begin{array}{l}\text { Between-study } \\
\text { variances }\left(\tau^{2}\right. \\
\text { seven levels })\end{array}$ & $0, .04, .08, .16, .24, .32$, and .50 & The effect size distributions assumed to be normal. \\
\hline $\begin{array}{l}\text { Distributions (six } \\
\text { levels) }\end{array}$ & $\begin{array}{l}\text { Normal (denoted as norm), negative exponential (or exponential, } \\
\text { positively skewed, denoted as exp), and reversed exponential } \\
\text { distributions (negatively skewed, denoted as rev) }\end{array}$ & There are six combinations of the four distributions. \\
\hline
\end{tabular}

${ }^{a}$ Because the sample sizes are equal for the two groups, which are $n_{1}=n_{2}, \delta_{i} \sqrt{\left[\left(n_{1}-1\right)+\left(n_{2}-1\right) S R_{i}^{2}\right] /\left(n_{1}+n_{2}-2\right)}$ can be simplified to $\delta_{i}$ $\sqrt{\left(S R_{i}^{2}+1\right) / 2}$ in this case.

collected meta-analyses in Psychological Bulletin from 1995 to 2005 , summarized 81 meta-analyses, and found that the 10th percentile of $k$ was 5 , the median was 76 , and the 90 th percentile was 133 .

Fourth, the between-study variance, $\tau^{2}$, was manipulated, with values of $0,0.04,0.08,0.16,0.24,0.32$, and 0.50 . These values have been common in previous simulations (e.g., Johnson \& Huedo-Medina, 2013; MarínMartínez \& Sánchez-Meca, 2010). We also included 0.24 and 0.50 , because the review conducted by Brannick, Yang, and Cafri (2011) showed that these values are the median and the 90th percentile, respectively, of estimated $\tau^{2}$ in applied psychology areas. If $\tau^{2}$ is greater than 0 , the population effect sizes were normally distributed with mean population effect sizes $\delta$ and variance equal to $\tau^{2}$. Once $\delta_{i}$ was generated in the $i$ th study, the data in the two groups were generated from the distributions described below. The population mean and standard deviation in one group were set to 0 and 1 , respectively. In the other group, the population standard deviation was set to $1,2,4,0.5$, and 0.25 , depending on the $S D$ ratio of this condition $\left(S R_{i}\right)$, and the population mean was fixed at $\delta_{i} \sqrt{\left(S R_{i}^{2}+1\right) / 2}$, ensuring that the corresponding population SMD was equal to $\delta_{i}$.

Fifth, the within-group distributions investigated were normal (denoted as norm below), exponential (denoted as exp below) and reversed exponential distributions (denoted as $r e v$ below). Random numbers from these distributions were generated by the built-in functions in R ( R Core Team, 2018). The arguments of these functions were specified and the generated numbers were rescaled such that the population means, population standard deviations, and population SMD are of the desired values. The skewness and excess kurtosis for the distributions are 0 and 0 for normal distribution, 2 and 6 for the exponential distribution, and -2 and 6 for the reversed exponential distribution, respectively. We have also examined uniform and logistic distributions in a preliminary simulation. Because there were negligible differences among these distributions (e.g., uniform-uniform, logistic-logistic) and normal distributions (normnorm) in the outcome measures, we do not report these conditions in the present article. Thus, there were six combinations of distributions for the two groups: norm-norm, exp-exp, exp-rev, rev-exp, exp-norm, and norm-exp. Examples of these combinations of distributions are shown in Fig. 1, with the population effect size equal to 1.4, the between-study variance equal to 0 , and the sample sizes equal to $80\left(n_{1}=n_{2}=80\right)$. The graphs also show the theoretical density plots of data simulated from norm-norm, exp-exp, norm-exp, exp-norm, rev-exp, and exp-rev..

In sum, the simulation study had 25,200 conditions: 6 (population effect size, $\delta) \times 5(S D$ ratio $) \times 4$ (mean sample size, $\bar{N}) \times 5($ study size, $k) \times 7$ (heterogeneity variance, $\left.\tau^{2}\right) \times 6$ (distribution combinations). Each condition had 5,000 replications.

\section{Meta-analytic procedures}

In the present study, we adopted only the random-effect model, which allowed the studies to have different 

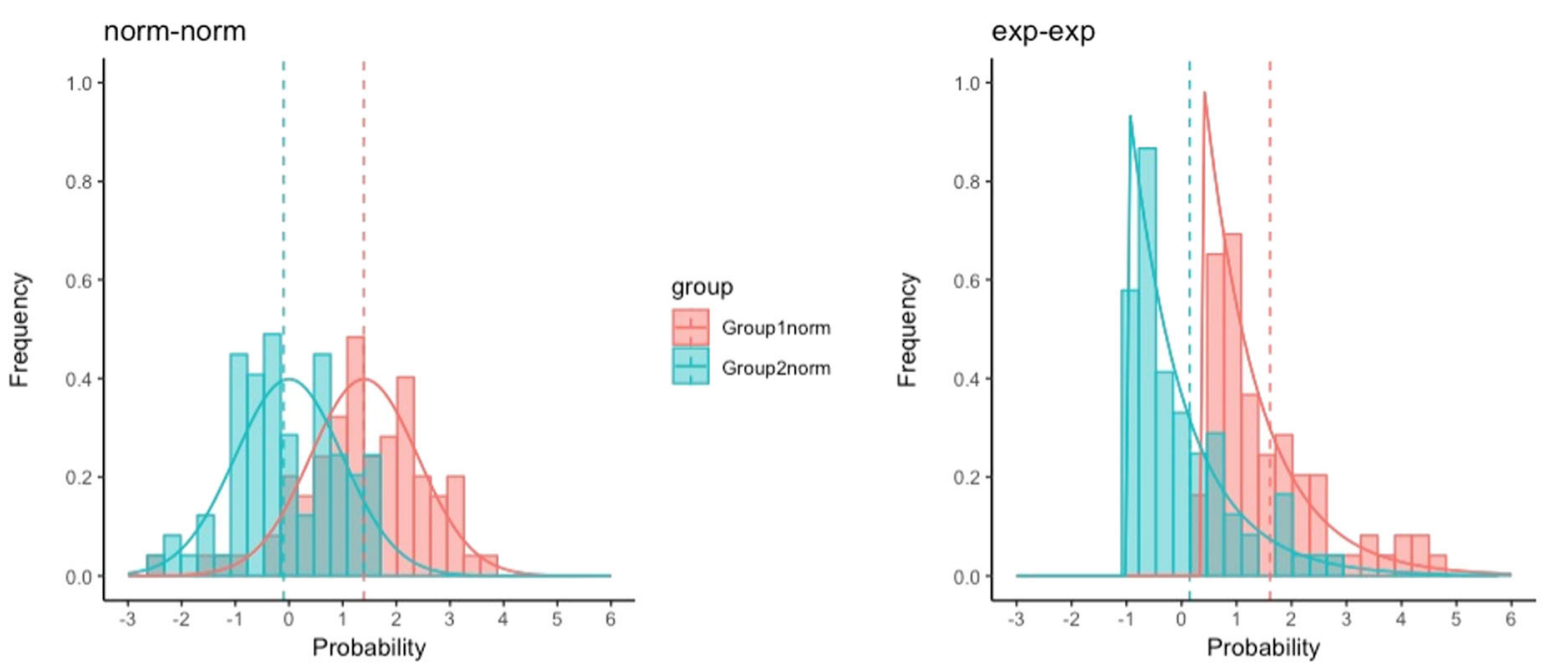

group

Group1exp

Group2exp
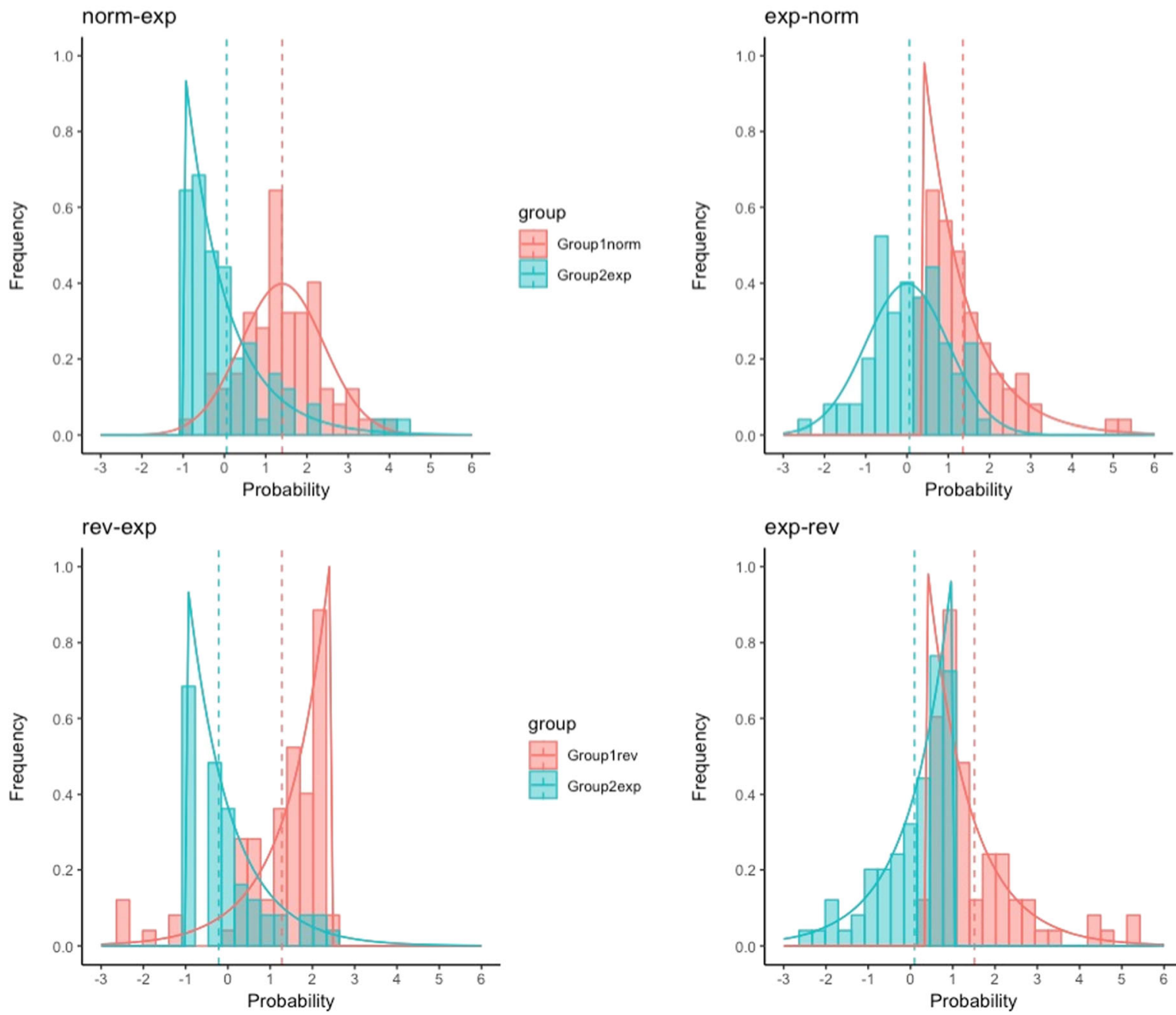

group
\begin{tabular}{|l|l}
\hline 1 & Group 1norm \\
\hline 1 & Group2exp
\end{tabular}

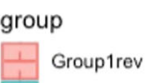

Group2exp

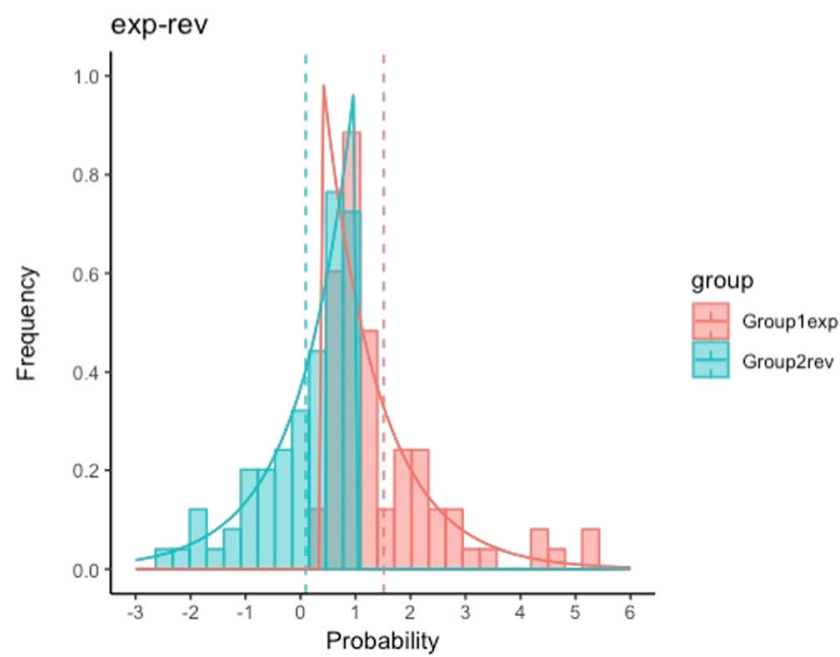

Fig. 1 Plots of empirical cumulative distribution functions when the population effect size is 1.4 , the between-study variance is 0 , the sample sizes in the two independent groups are 80 , and the $S D$ ratio is equal to 1

population effect sizes, for two reasons. First, in many meta-analyses, this model is much more plausible than the fixed-effect model, which assumes that all studies

(homoscedasticity, equal variance), with the theoretical density curves overlaid. The two vertical lines are the population means of the two groups

have the same population effect size. Second, the random-effect model is much more popular in metaanalyses than fixed-effect model. 
We applied the DerSimonian-Laird (DL) procedure in order to combine effect size estimates (DerSimonian \& Laird, 1986). First, $Q$ is used in the $\chi^{2}$ homogeneity test and is computed by

$Q=\sum_{i=1}^{k} \frac{\left(g_{i}-g_{w}\right)^{2}}{v_{i}}$

where

$g_{w}=\frac{\sum_{i=1}^{k} w_{i} g_{i}}{\sum_{i=1}^{k} w_{i}}$,

$g_{i}$ is the Hedges's $g$ of the $i$ th study, and $w_{i}$ is equal to $1 / v_{i}, v_{i}$ being the estimate of sampling variance of $g_{i}$ for the $i$ th study (computed by Eq. 4). The heterogeneity variance estimator in the DL procedure is based on the method of moment and is estimated from $Q$ as below (DerSimonian \& Laird, 1986),

$\hat{\tau}_{D L}^{2}=\frac{Q^{-}(k-1)}{c}$, if $Q>k^{-1}$,

and $c$ is equal to

$c=\sum_{i=1}^{k} w_{i}-\frac{\sum_{i=1}^{k} w_{i}^{2}}{\sum_{i=1}^{k} w_{i}}$.

If $Q$ is less than $k-1, \uparrow_{D L}^{2}$ is truncated to zero.

The mean population effect size is then estimated, taking into account both the sampling variance and the betweenstudy variance. The weighting in random-effect model for the effect size from the $i$ th study, $w_{i}^{*}$, is the inverse of the estimated total variance $\partial^{2}\left(g_{i}\right)$, which is equal to $\widehat{T}_{D L}^{2}$ plus $v_{i}$ (DerSimonian \& Laird, 1986; Hedges, 1983; Hedges \& Olkin, 1985; Hedges \& Vevea, 1998):

$w_{i}^{*}=\frac{1}{\hat{\sigma}^{2}\left(g_{i}\right)}=\frac{1}{\hat{\tau}_{D L}^{2}+v_{i}}$.

The mean effect size in random-effect model, $g_{D L}^{*}$, is then given by (Hedges, 1981, 1982, 1983)

$g_{D L}^{*}=\frac{\sum_{i=1}^{k} w_{i}^{*} g_{i}}{\sum_{i=1}^{k} w_{i}^{*}}$

and its sampling variance, $v_{+}^{*}$, is given by

$v_{+}^{*}=\frac{1}{\sum_{i=1}^{k} w_{i}^{*}}$.

The $95 \%$ confidence interval for the mean effect size is given by $g_{D L}^{*} \pm 1.96 * \sqrt{v_{+}^{*}}$ (Hedges \& Vevea, 1998).

\section{Outcome measures}

Bias and mean squared error (MSE) To assess the accuracy and efficiency of an estimator, the bias and MSE were computed. The bias for estimating the population mean effect size $\delta$ was computed as

$\operatorname{BIAS}(\hat{\partial})=\frac{\sum g_{+j}}{n r e p}-\delta$,

where nrep is the number of replications and $\delta$ is the population effect size of $j$ th replication.

The MSE is the mean squared difference between the parameter (e.g., $\delta$ ) and the estimate (Marín-Martínez \& SánchezMeca, 2010). It is given by

$\operatorname{MSE}(\hat{\partial})=\frac{\sum\left(g_{+j}-\delta\right)^{2}}{\text { nrep }}$.

Estimators that show small bias and MSE are preferred by researchers.

Coverage probability of confidence interval The coverage probability of the confidence interval assesses the extent that the confidence interval captures the population effect size in a meta-analysis as often as expected. It is given by

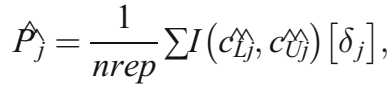

where $\hat{c_{L} j}$ and $\hat{c}_{U j}$ are the lower and upper confidence interval limits, respectively, in the $j$ th replication, and

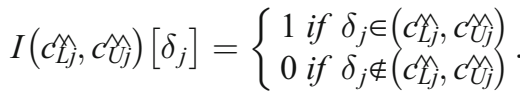

The nominal coverage probability for a $95 \%$ confidence interval is .95.

\section{Results}

To compare the differences among different distributions, we assessed the bias, MSE, and estimated coverage probability of the mean effect sizes in a metaanalysis across the 5,000 replications of each condition. Also, we computed the Type I error rates and empirical power rates of the $Q$ tests. Due to space limitations, we averaged the bias, MSE, estimated coverage probability, Type I error rates, and empirical power of the $Q$ tests across selected manipulated factors as appropriate for each section. 


\section{Bias and MSE in estimating mean effect sizes}

Figure 2 shows a comparison of norm-norm, exp-exp, exprev, rev-exp, exp-norm, and norm-exp in terms of average bias. It shows clearly that the mean effect sizes in metaanalytic results could be exaggerated (overestimated) due to nonnormal data, particularly when the distributions are oppositely skewed (e.g., rev-exp). Additionally, the directions of the tails influence the results: When the tails were pointing away from each other's means (i.e., rev-exp and norm-exp), in most conditions the biases were positive; when the tails were pointing toward each other's means (i.e., exp-rev and exp-norm), the biases were usually negative. The average bias values, computed through all the conditions, for norm-norm was -.012 , for exp-exp was .024, for exp-rev was - .023, for rev-exp was .076, for exp-norm was - .014, and for normexp was .032. When the distributions were norm-norm, exprev, and exp-norm, all manipulated parameters seemed to have little influence on the negligible values of bias. When the distributions were exp-exp and norm-exp, the average bias tended to decrease in magnitude with an increase in $S D$ ratio, a decrease in population effect size, and an increase in average sample size. The positive bias of the rev-exp condition was most noticeable compared with the other distributions. Also, the study size and between-study variance scarcely affected the bias in all distribution conditions.

Figure 3 shows a comparison of norm-norm, exp-exp, exp-rev, rev-exp, exp-norm, and norm-exp in terms of MSE. Similar to the simulation by Marín-Martínez and Sánchez-Meca (2010), MSE increased as the study size and sample size decreased and as the between-study variance increased. Moreover, MSE tended to increase as the SD ratio decreased. When the distributions were rev-exp, norm-exp, and exp-exp, the MSE tended to increase as the population effect size increased. The rev-exp conditions showed the largest MSE compared with the other distributions, across conditions. The results from Fig. 2 suggest that this is due to the large positive bias in the rev-exp conditions. For example, in meta-analyses with between-study variance equal to 0.08 , study size equal to 20 , average sample size equal to $50, S D$ ratio equal to 1 , and population effect size equal to 0.5 , the $M S E$ was .0043 for norm-norm, .0049 for exp-exp (relative
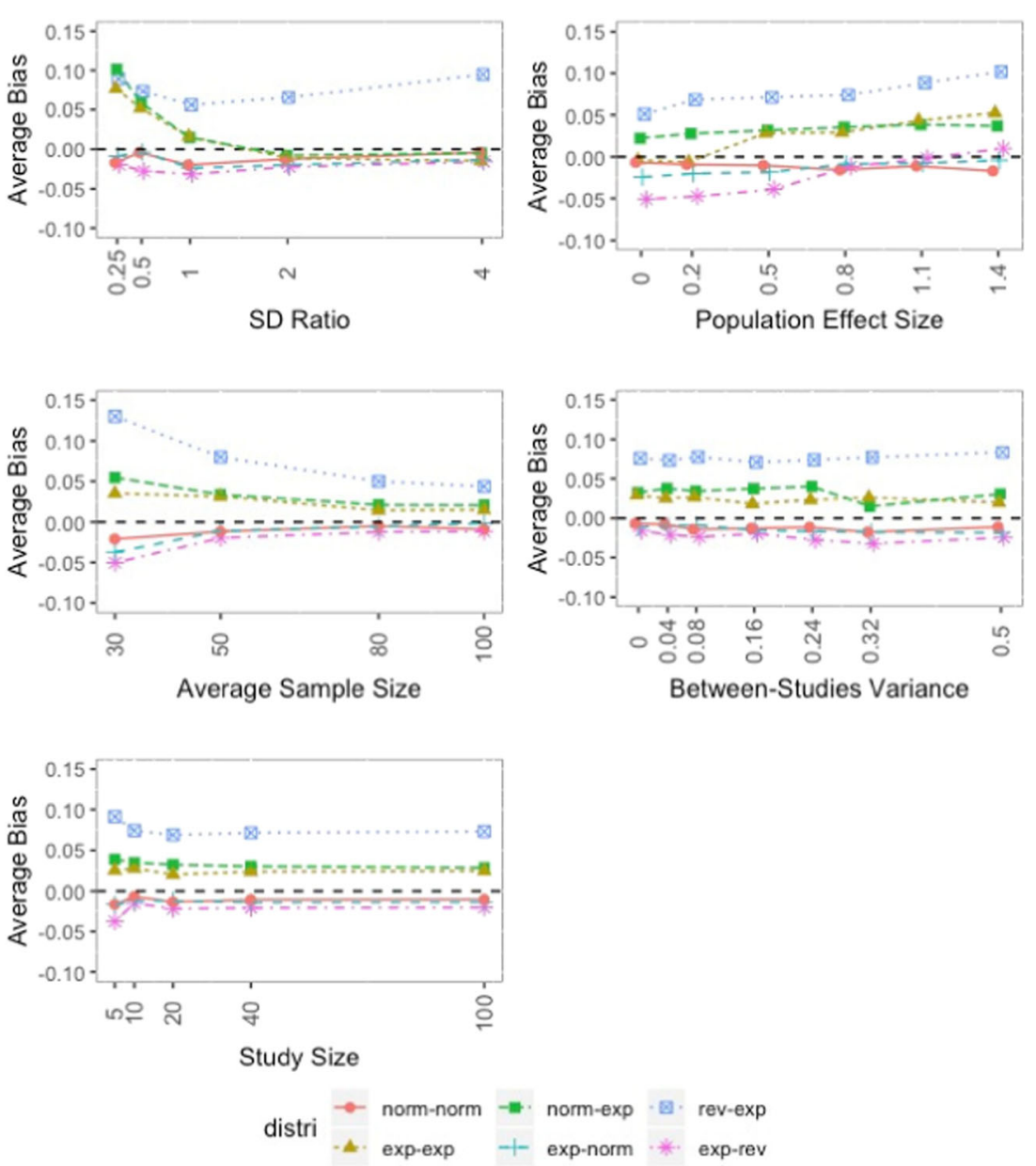

Fig. 2 Average bias values as a function of the $S D$ ratio, population effect size, average sample size, between-study variance, and study size under six distribution combinations 

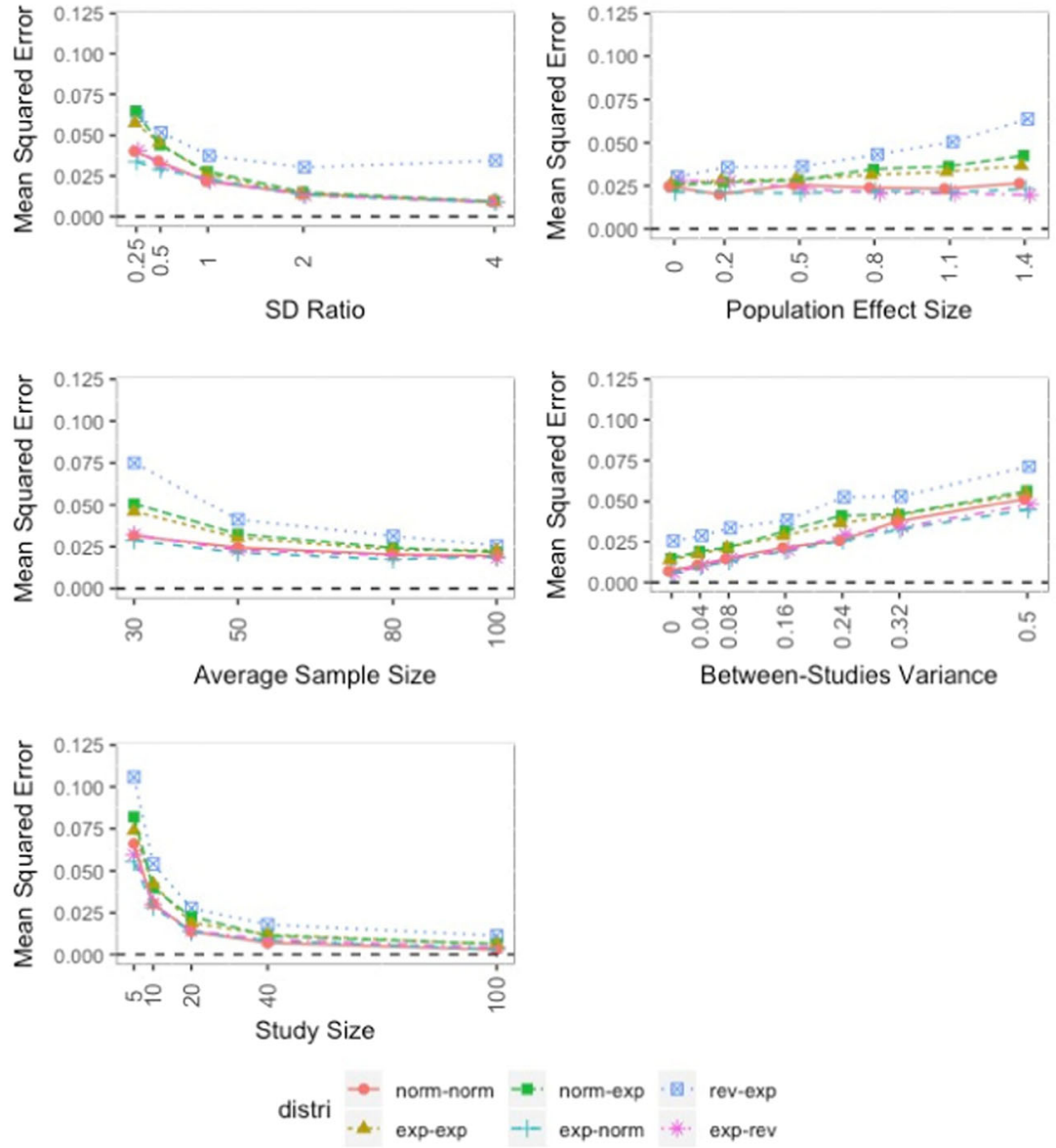

Fig. 3 Average mean squared error values as a function of the $S D$ ratio, population effect size, average sample size, between-study variance, and study size under six distribution combinations

increase of $13.95 \%$ with respect to .0043$), .0061$ for norm$\exp$ (relative increase of $41.85 \%$ with respect to .0043 ), .0058 for exp-norm (relative increase of $34.88 \%$ with respect to .0043 ), .0114 for rev-exp (relative increase of $165.12 \%$ with respect to .0043), and .0041 for exp-rev (relative decrease of $4.65 \%$ with respect to .0043 ). The average $M S E$ values for the different distributions across all conditions were .0240 for norm-norm, .0306 for exp-exp, .0323 for norm-exp, .0217 for exp-norm, .0433 for rev-exp, and .0233 for exp-rev. When the tails pointed toward each other's means, the mean effect sizes in the meta-analysis were the least efficient across conditions.

\section{Coverage probability for mean effect sizes}

To assess the influence of the manipulated factors, we applied Bradley's (1978) liberal criterion. By this criterion, Type I error rates within .025 of the nominal value are acceptable. This range corresponds to coverage probabilities within .925 and .975 for a $95 \%$ confidence interval. Figure 4 depicts the average coverage probabilities for the mean effect sizes as a function of $S D$ ratio, population effect size, average sample size, between-study variance, and study size. Overall, the nonnormal data deviated more from the nominal value of .95 than did the normal data, especially in the conditions of oppositely skewed distributions (e.g., rev-exp). The patterns were similar to those for bias in Fig. 2.

For the norm-norm conditions, the average coverage probabilities were slightly below the nominal level but were inside the acceptable range by Bradley's criterion. However, when the study size was extremely small $(k=5)$ or the betweenstudy variances were large $\left(\tau^{2} \geq .32\right)$, the average coverage probabilities were outside the acceptable range. In general, the empirical coverages got closer to the nominal level for large $S D$ ratio, small between-study variance, and large study size when the distribution was norm-norm. However, for all conditions with at least one nonnormal distribution, larger study sizes always led to greater deviation from the nominal level, particularly for rev-exp. Also, when the distribution was rev-exp, the average coverage probabilities got closer to the nominal level only when the $S D$ ratio got closer to 1 - that is, when the population standard deviations of the two groups were equal. For exp-exp and norm-exp, a smaller $S D$ ratio led to average coverage probabilities that deviated from the 

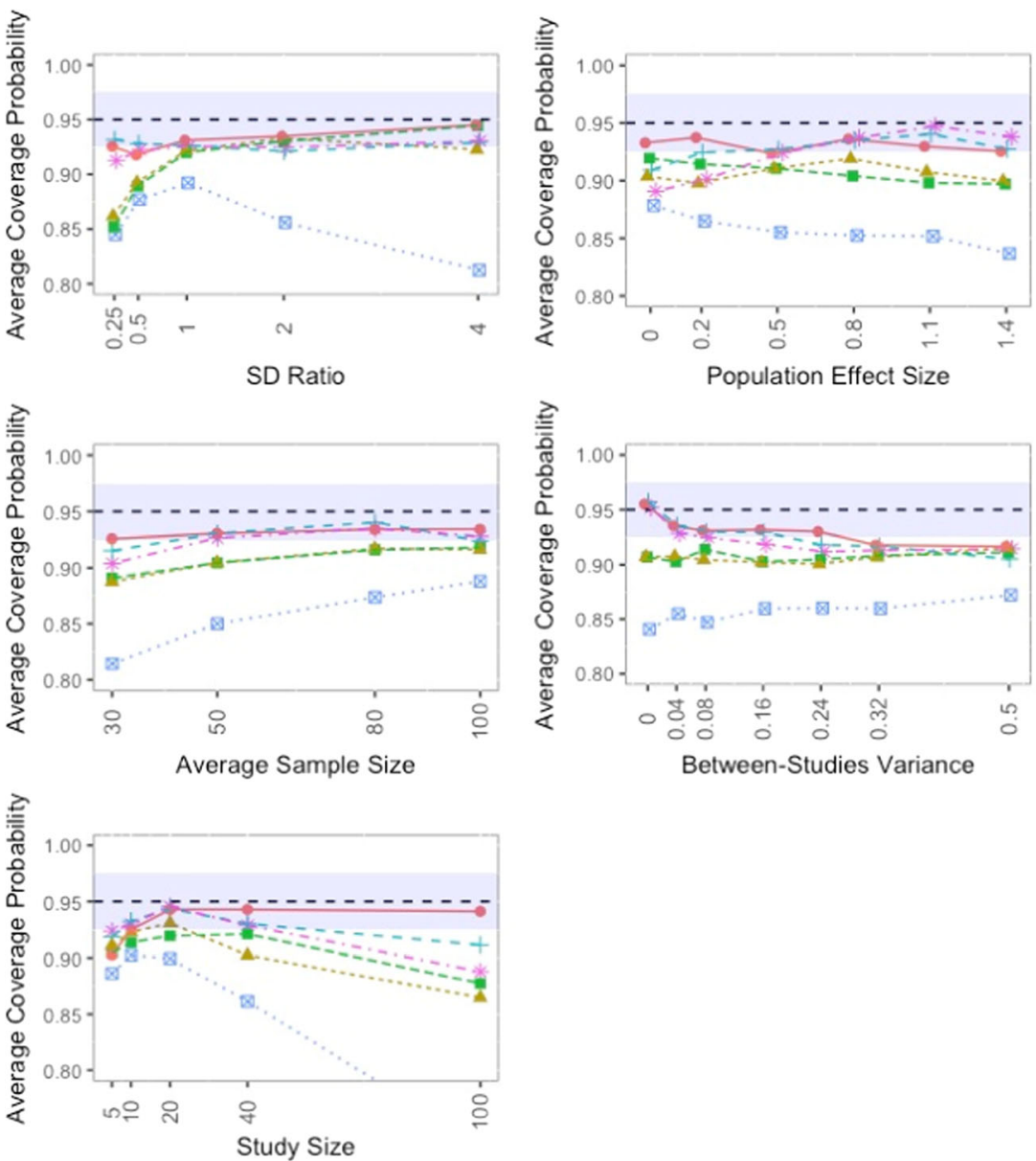

Between-Studies Variance

distri

- norm-norm - norm-exp $\cdot$ - 0 rev-exp
4. exp-exp + exp-norm * exp-rev

Fig. 4 Average coverage probabilities as a function of the $S D$ ratio, population effect size, average sample size, between-study variance, and study size under six distribution combinations. The shaded areas

are within the criterion range of .925 to .975 . The horizontal dotted lines (black) indicate the nominal coverage probability $(.95)$

nominal level. Additionally, the larger the population effect size, the larger the difference in average coverage probabilities between exp-rev and rev-exp, as well as between norm-exp and exp-norm.

\section{Type I error rate and power of the $\mathbf{Q}$ test}

For each condition, the rejection rate of the $Q$ test refers to the proportion of replications with significant test results in this condition. The Type I error rate of the $Q$ test refers to the rejection rate in conditions in which the between-study variance was equal to zero $\left(\tau^{2}=0\right)$. The power of the $Q$ test refers to the same rejection rate, but in conditions in which the between-study variance is greater than zero $\left(\tau^{2}>0\right)$. Figure 5 presents the Type I error rates of the $Q$ test as a function of $S D$ ratio, population effect size, sample size, and study size under different distributions. As in the previous study by Viechtbauer (2007), the Type I error rate of the $Q$ test showed little dependence on the population effect sizes for normal

distributions (norm-norm), as well as for the exp-norm and exp-rev conditions. However, for norm-exp, rev-exp, and exp-exp, larger population effect sizes led to Type I error rates higher than the nominal level. This result is different from those in the simulations by Harwell (1997) and HuedoMedina et al. (2006), in which skewed distributions had little effect on the performance of the $Q$ test in their studies.

To further explore those factors, we selected the conditions in which the $S D$ ratio was equal to 1 and 0.5 (shown in Figs. 6 and 7, respectively), because the patterns when $S D$ ratios were equal to 1,2, and 4 were similar, and the patterns between $S D$ ratio $=0.25$ and $S D$ ratio $=0.5$ were similar. The figures show clearly that an increase in population effect size led to an increase in Type I error rates for rev-exp and norm-exp, resulting in Type I error rates as high as $90 \%$ in some revexp conditions. The divergence of this result from those of previous simulations comes from the distributions selected for data generation. Even though the simulations by Harwell (1997) included exponential distributions, Harwell only 

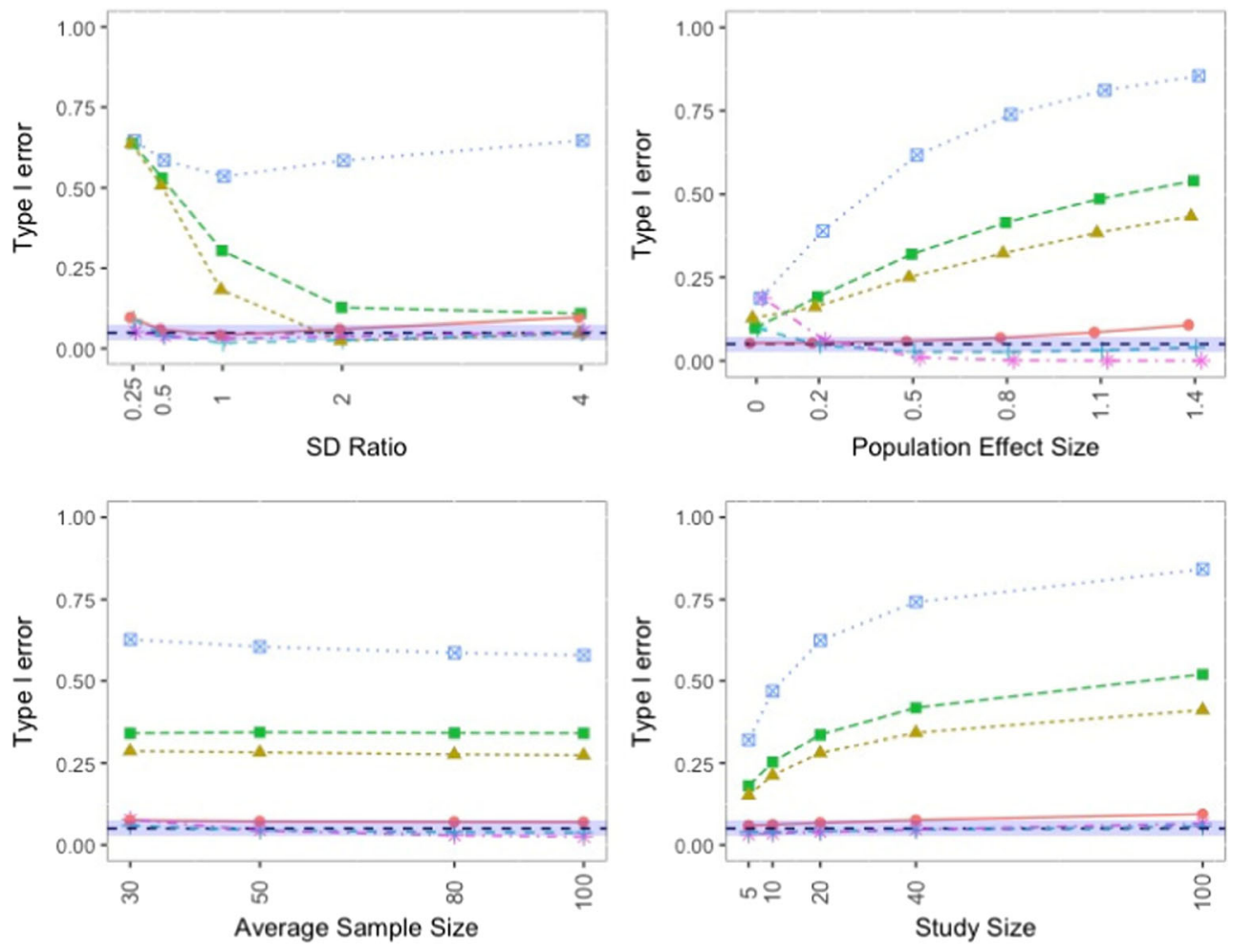

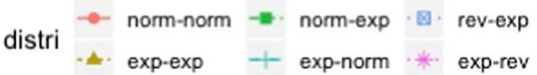

Fig. 5 Type I error rates of $Q$ tests as a function of $S D$ ratio, population effect size, average sample size, and study size under different distributions

simulated the same distributions for the two groups. Similarly, Huedo-Medina et al. (2006) studied groups with the same distribution shapes and found little effect of population effect sizes on the performance of the $Q$ test.

Furthermore, Fig. 7 also supports the result of HuedoMedina et al. (2006) that nonnormality and increased $S D$ ratio together drastically increase the Type I error rate. When the $S D$ ratio was equal to 0.5 , even for conditions with the same nonnormal distributions - that is, the exp-exp conditionsType I error rates increased with increasing population effect sizes. Additionally, as in previous studies (Harwell, 1997; Huedo-Medina et al., 2006; Viechtbauer, 2017), small sample size paired with large study size could result in inflated Type I error rates.

One thing that should be borne in mind is that the actual Type I error rates influence the empirical power of the $Q$ test. In this way, tests with higher Type I error rates, in turn, showed higher probability to detect heterogeneity when there was heterogeneity in the population effect sizes. There was little difference among other distribution combinations, except for rev-exp, whose rejection rates were higher than in normal conditions. Also, increasing the sample size, study size, or between-study variance, and decreasing the $S D$ ratio led to higher probabilities of rejecting the null hypothesis (Van den Noortgate \& Onghena, 2003; Viechtbauer, 2017).

\section{Discussion}

In meta-analysis, researchers are rarely concerned about nonnormality in the primary studies, and thus it is not clear how nonnormality of the raw data influences meta-analytic results. To fill this research gap, we simulated various scenarios to examine selected forms of nonnormal data. Our simulation examined the bias, MSE, and coverage probability of the confidence interval in estimating the mean population effect size, and also the performance of the $Q$ test. The metaanalytic procedure - the DL procedure (DerSimonian \& Laird, 1986) - that we applied is one of the most popular procedures and is introduced in many meta-analysis books (e.g., Card, 2015; Cooper, Hedges, \& Valentine, 2009). The present study was the first to examine oppositely skewed distributions in meta-analysis using a Monte Carlo simulation. We also have provided a database of simulation results, to give guidelines for researchers who are interested in particular conditions in a given area (the database can be downloaded from the website http://osf.io/m5cze/?view only= 19d01aefb26444ad8156dd306b1c5272).

Practically, it is important to explore skewed distributions, particularly exponential distributions. First, as we mentioned above, exponential distributions are the distributions conventionally examined in Monte Carlo simulations of nonnormal 


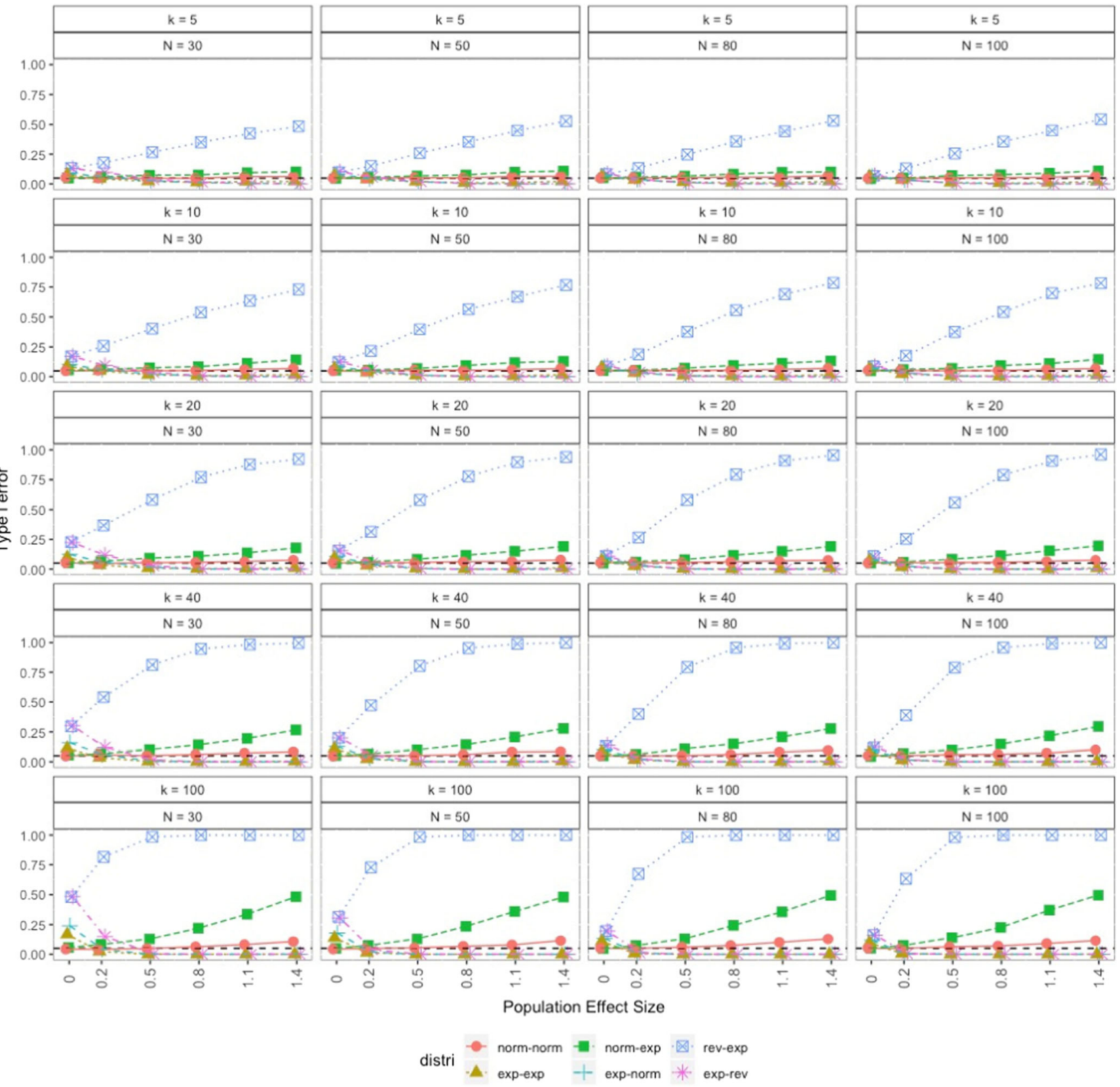

Fig. 6 Type I error rates of $Q$ tests when the $S D$ ratio is equal to 1

data (e.g., Harwell et al., 1992; Keselman et al., 2005). Second, exponential distributions have practical meaning and can be applied in order to model response time, physical and verbal violence in couples, and similar variables (see more examples in Bono et al., 2017). Third, it is essential to explore exponential distribution with normal distributions (exp-norm and norm-exp conditions) because it can model the floor effect, which can often happen in behavioral research when one group outperforms the other group (e.g., the comparisons between schizophrenic patients and a control group by Leentjens et al., 1998). We did not include a rev-norm condition, because it is a mirror image of norm-exp. However, from the results for norm-exp, we could tell the effect of rev-norm in primary studies on meta-analytic results, suggesting similar problems in the study with the ceiling effect in one group.

The main contribution of this article is that we found that the positions of the skewed distributions of the two groups influence the results. As in previous simulations (e.g., Johnson \& Huedo-Medina, 2013), nonnormality could increase bias and MSE, especially with nonhomomerity (skewed in different directions). Our results also extended the results from previous studies of nonnormality and nonhomomerity with other tests (e.g., Welch's $F$ test: Cribbie et al., 2012; $t$ test: Delaney \& Vargha, 2000; Tiku, 1964), that oppositely 


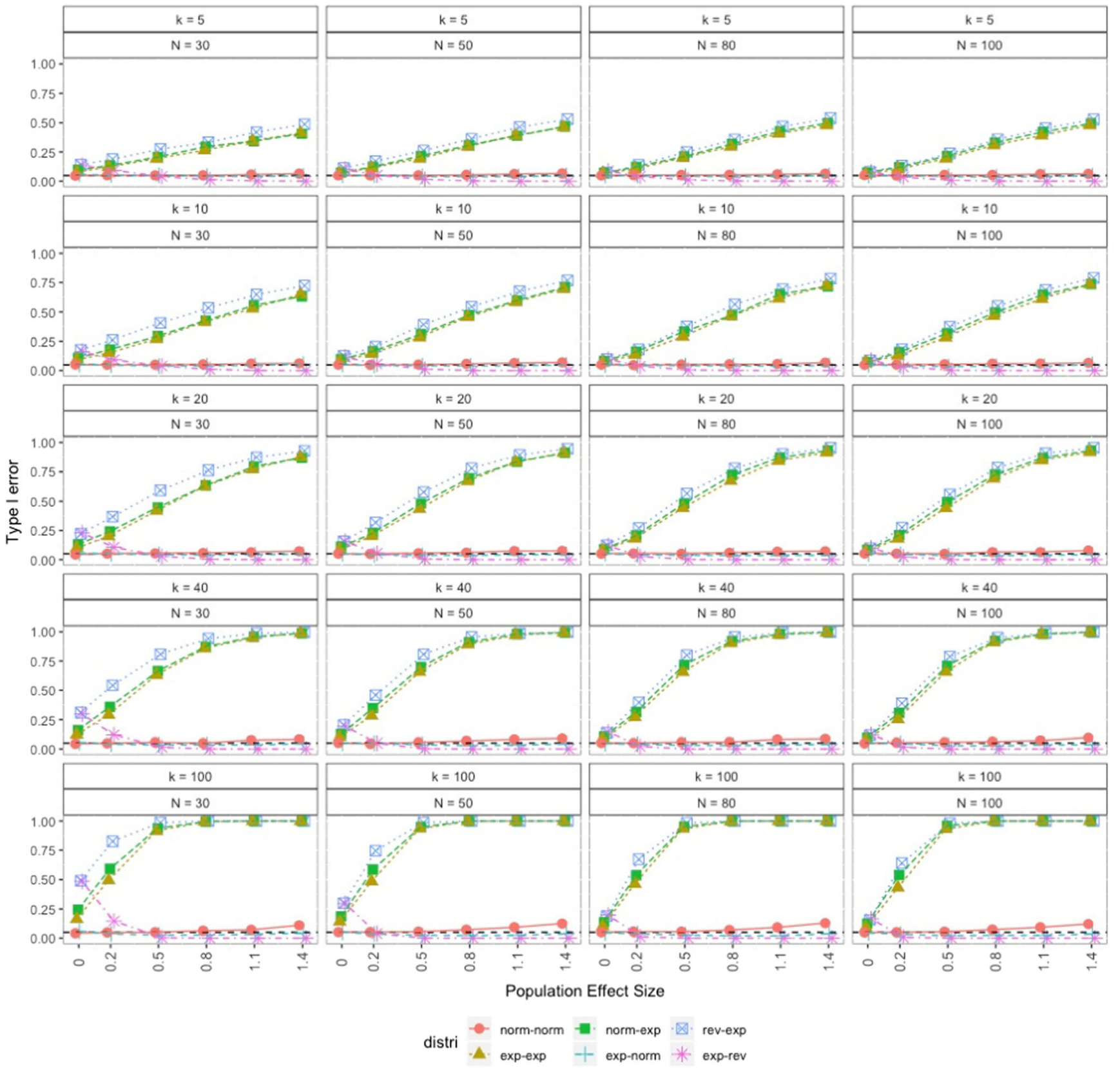

Fig. 7 Type I error rates of $Q$ tests when the $S D$ ratio is equal to 0.5

skewed distributions in the primary studies lead to less accurate point estimates of mean effect size and suboptimal coverage probability of interval estimates of the mean effect size in a meta-analysis. We further found that, though the exprev and rev-exp conditions are both oppositely skewed distributions, the bias, MSE, and coverage probability of mean effect sizes in a meta-analysis are substantially different for the two conditions. The exp-rev condition showed positive bias under most conditions, larger $M S E$, and lower estimated coverage, whereas rev-exp showed negative bias under most conditions, smaller $M S E$, and higher estimated coverage.
Another contribution is that we found an influence of nonhomomerity on the performance of the $Q$ test. Previous simulations on meta-analyzing SMD had only investigated scenarios in which the distribution shapes for the two groups in SMD were the same. However, this study showed that oppositely skewed distributions, particularly rev-exp and norm-exp, tended to produce inflated Type I error rates for the homogeneity test. This was not the case for the normnorm, exp-rev, and exp-norm conditions. Our findings, combined with previous results, show a more comprehensive picture of the impact of nonnormal data on the $Q$ test. Also, we found that rev-exp and norm-exp also had higher 
probabilities of rejecting the null hypothesis than did exp-rev and exp-norm. Considering the too-high Type I error rates under those conditions, this does not mean that it is easier to detect heterogeneity. It means that homogeneity tests cannot distinguish nonhomomerity of the distributions from heterogeneity in the population SMDs (or the presence of moderators).

It is not yet clear why the direction of a skewed distribution could influence the meta-analytic results. We suspect that the long tail of skewed distribution causes this problem. One piece of evidence to support this point is that under the norm-exp and exp-exp conditions, the relation of average bias to $S D$ ratio was monotonic: That is, the bias decreased when the $S D$ ratio increased. But under the rev-exp condition, the relation between average bias and $S D$ ratio was not monotonic: The bias decreased with increased $S D$ ratio when the $S D$ ratio was smaller than 1 , and the bias increased with increased $S D$ ratio when the $S D$ ratio was larger than 1 . Because the standard deviation of the first group was equal to the $S D$ ratio and the standard deviation of the second group was set to 1 , the larger $S D$ ratio means a larger standard deviation of the first group. The differences in the relation of average bias and $S D$ ratio between norm-exp, exp-exp, and rev-exp might come from the different effects of increasing standard deviation in the tails of the normal (norm), exponential (exp), and reversed exponential (rev) distributions.

Our study has four limitations. First, even though we explored six combinations of distributions, it was impossible to examine every possible combination of nonnormal distributions. More studies will be needed to examine how other kinds of commonly found nonnormal distributions might affect meta-analytic results. Second, we investigated nonnormality in the population raw data in the primary studies. In reality, such nonnormality could be due to contamination, measurement error, and other study-level factors, resulting in betweenstudy variation in the distributions of the raw data. Future studies can investigate how the composition of various types of distributions in a meta-analysis, such as some studies being norm-norm while some are norm-exp, might affect the metaanalytic results. Third, the distribution of effect sizes in our simulation was assumed to be normal in the simulation. However, this assumption might not be tenable in some situations (Baker \& Jackson, 2008). Further studies can investigate how nonnormal random effects can affect the metaanalytic results. Finally, although we considered a broad range of sample sizes, we did not consider the conditions with unequal sample sizes within the individual study (i.e., $n_{1 i} \neq, n_{2 i}$ ). Further studies can examine the more complicated situations in which two groups have unequal sample sizes.

In addition to the limitations of our simulation study mentioned above, several directions are suggested for future studies. There are other commonly used meta-analytic procedures, particularly robust meta-analysis (e.g., Bonett, 2009), on which nonnormality and nonhomomerity might have effects different from those we found. A comparison among different meta-analytic procedures in the presence of nonnormal data could guide researchers on the selection of meta-analytic procedures. Furthermore, Bayesian methods are becoming more and more popular. To the extent that a normal distribution is assumed in the data generation process, the impact of nonnormal populations on Bayesian meta-analysis also needs to be examined.

In summary, three conclusions can be drawn from our study. First, skewed distributions with tails pointing toward each other can cause exaggerated and distorted point and interval estimates of the mean SMD in meta-analysis. In practice, we recommend that meta-analysts report the mean, standard deviation, and maximum and minimum possible values of the measure in each group for the SMDs being summarized. These values were usually reported in the primary studies, and one could identify probable ceiling or floor effects on the basis of this information. They can also help readers judge to what extent results might be affected by nonnormality in the raw data. Second, the results of $Q$ tests under oppositely skewed conditions (rev-exp and norm-exp) should be interpreted with caution. Even without raw data, in some conditions it will be reasonable to suspect that the data in the two groups are oppositely skewed (e.g., one group is expected to have a ceiling or floor effect in the distribution). Those conditions (rev-exp and norm-exp) could result in substantially inflated Type I error rates for the $Q$ test. Third, even though there is no simple solution to address nonnormal data in meta-analysis, because the raw data are rarely available for primary studies, caution is required when meta-analyzing variables that theoretically are likely to be skewed. The violation of normality should not be ignored, even in meta-analysis.

Author Note This research received no specific grant from any funding agency in the public, commercial, or not-for-profit sectors.

Open Practice Statement The simulation data and supplemental materials related to this article are available at http://osf.io/ m5cze/?view_only=19d01aefb26444ad8156dd306b1c5272.

\section{References}

Algina, J., Keselman, H. J., \& Penfield, R. D. (2005). An alternative to Cohen's standardized mean difference effect size: A robust parameter and confidence interval in the two independent groups case. Psychological Methods, 10, 317-328. https://doi.org/10.1037/ 1082-989X.10.3.317

Algina, J., Keselman, H. J., \& Penfield, R. D. (2006). Confidence interval coverage for Cohen's effect size statistic. Educational and 
Psychological Measurement, 66, 945-960. https://doi.org/10.1177/ 0013164406288161

Baker, R., \& Jackson, D. (2008). A new approach to outliers in metaanalysis. Health Care Management Science, 11, 121-131. https:// doi.org/10.1007/s10729-007-9041-8

Bendayan, R., Arnau, J., Blanca, M. J., \& Bono, R. (2013). Comparison of the procedures of Fleishman and Ramberg et al. for generating non-normal data in simulation studies. Annals of Psychology, 30, 364-371. https://doi.org/10.6018/analesps.30.1.135911

Blanca, M. J., Arnau, J., López-Montiel, D., Bono, R., \& Bendayan, R. (2013). Skewness and kurtosis in real data samples. Methodology, 9 , 78-84. https://doi.org/10.1027/1614-2241/a000057

Bonett, D. G. (2008). Confidence intervals for standardized linear contrasts of means. Psychological Methods, 13, 99-109. https://doi.org/ 10.1037/1082-989X.13.2.99

Bonett, D. G. (2009). Meta-analytic interval estimation for standardized and unstandardized mean differences. Psychological Methods, 14, 225-238. https://doi.org/10.1037/a0016619

Bono, R., Blanca, M. J., Arnau, J., \& Gómez-Benito, J. (2017). Nonnormal distributions commonly used in health, education, and social sciences: A systematic review. Frontiers in Psychology, 8, 1602. https://doi.org/10.3389/fpsyg.2017.01602

Bradley, J. V. (1978). Robustness? British Journal of Mathematical and Statistical Psychology, 31, 144-152. https://doi.org/10.1111/j.20448317.1978.tb00581.x

Brannick, M. T., Yang, L.-Q., \& Cafri, G. (2011). Comparison of weights for meta-analysis of $r$ and $d$ under realistic conditions. Organizational Research Methods, 14, 587-607. https://doi.org/ $10.1177 / 1094428110368725$

Cafri, G., Kromrey, J. D., \& Brannick, M. T. (2010). A meta-meta-analysis: Empirical review of statistical power, type I error rates, effect sizes, and model selection of meta-analyses published in psychology. Multivariate Behavioral Research, 45, 239-270. https://doi.org/ 10.1080/00273171003680187

Card, N. A. (2015). Applied meta-analysis for social science research. New York, NY: Guilford Press.

Cohen, J. (1988). Statistical power analysis for the behavioral sciences. Hillsdale, NJ: Erlbaum.

Cooper, H., Hedges, L. V., \& Valentine, J. C. (Eds.). (2009). The handbook of research synthesis and meta-analysis. Washington, DC: Russell Sage Foundation.

Cribbie, R. A., Fiksenbaum, L., Keselman, H., \& Wilcox, R. R. (2012). Effect of non-normality on test statistics for one-way independent groups designs. British Journal of Mathematical and Statistical Psychology, 65, 56-73. https://doi.org/10.1111/j.2044-8317.2011. 02014.x

Delaney, H. D., \& Vargha, A. (2000, April). The effect of nonnormality on Student's two-sample t test. Paper presented at the Annual Meeting of the American Educational Research Association, New Orleans, LA.

DerSimonian, R., \& Laird, N. (1986). Meta-analysis in clinical trials. Controlled Clinical Trials, 7, 177-188. https://doi.org/10.1016/ 0197-2456(86)90046-2

Feingold, A. (1992). Sex differences in variability in intellectual abilities: A new look at an old controversy. Review of Educational Research, 62, 61-84. https://doi.org/10.3102/00346543062001061

Games, P. A., \& Hedges, L. V. (1987). Multifactor analyses on proportions, variances, correlations, and standardized mean differences for independent groups. Journal of Experimental Education, 56, 15-23. https://doi.org/10.1080/00220973.1987.10806461

Games, P. A., Keselman, H. J., \& Clinch, J. J. (1979). Tests for homogeneity of variance in factorial designs. Psychological Bulletin, 86, 978-984. https://doi.org/10.1037/0033-2909.86.5.978

Grissom, R. J., \& Kim, J. J. (2001). Review of assumptions of problems in the appropriate conceptualization of effect size. Psychological Methods, 6, 135-146. https://doi.org/10.1037/1082-989X.6.2.135
Harwell, M. (1997). An empirical study of Hedge's homogeneity test. Psychological Methods, 2, 219-231. https://doi.org/10.1037/1082989X.2.2.219

Harwell, M. R., Rubinstein, E. N., Hayes, W. S., \& Olds, C. C. (1992). Summarizing Monte Carlo results in methodological research: The one-and two-factor fixed effects ANOVA cases. Journal of educational statistics, 17, 315-339. https://doi.org/10.3102/ 10769986017004315

Hedges, L. V. (1981). . Journal of Educational and Behavioral Statistics, 6, 107-128. https://doi.org/10.3102/10769986006002107

Hedges, L. V. (1982) Fitting categorical models to effect sizes from a series of experiments. Journal of Educational Statistics, 7, 119-137. https://doi.org/10.2307/1164961

Hedges, L. V. (1983). A random effects model for effect sizes. Psychological Bulletin, 93, 388-395. https://doi.org/10.1037/00332909.93.2.388

Hedges, I. V., \& Olkin, I. (1985). Statistical methods for meta-analysis. San Diego, CA: Academic Press.

Hedges, L. V, \& Vevea, J. L. (1998). Fixed-and random-effects models in meta-analysis. Psychological Methods, 3, 486-504. https://doi.org/ 10.1037/1082-989X.3.4.486

Hess, M. R., \& Kromrey, J. D. (2004, April). Robust confidence intervals for effect sizes: A comparative study of Cohen's $d$ and Cliff's delta under non-normality and heterogeneous variances. Paper presented at the Annual Meeting of the American Educational Research Association, San Diego, CA.

Huedo-Medina, T. B., Jo, F., \& Botella, J. (2006). Assessing heterogeneity in meta-analysis: $Q$ statistic or $I^{2}$ index? Psychological Methods, 11, 193-206. https://doi.org/10.1037/1082-989X.11.2.193

Johnson, B. T., \& Huedo-Medina, T. B. (2013). Meta-analytic statistical inferences for continuous measure outcomes as a function of effect size metric and other assumptions. (AHRQ Publication No. 13EHC075-EF). Retrieved from https://www.ncbi.nlm.nih.gov/ books/NBK140575/pdf/Bookshelf_NBK140575.pdf

Joo, H., Aguinis, H., \& Bradley, K. J. (2017). Not all nonnormal distributions are created equal: Improved theoretical and measurement precision. Journal of Applied Psychology, 102, 1022-1053. https:// doi.org/10.1037/ap10000214

Kelley, K. (2005). The effects of nonnormal distributions on confidence intervals around the standardized mean difference: Bootstrap and parametric confidence intervals. Educational and Psychological Measurement, 65, 51-69. https://doi.org/10.1177/ 0013164404264850

Keselman, H. J., Algina, J., \& Fradette, K. (2005). Robust confidence intervals for effect size in the two-group case. Journal of Modern Applied Statistical Methods, 4, 353-371. https://doi.org/10.22237/ jmasm/1130803320

Leentjens, A. F., Wielaert, S. M., van Harskamp, F., \& Wilmink, F. W. (1998). Disturbances of affective prosody in patients with schizophrenia: A cross sectional study. Journal of Neurology, Neurosurgery and Psychiatry, 64, 375-378. https://doi.org/10. 1136/jnnp.64.3.375

Marín-Martínez, F., \& Sánchez-Meca, J. (2010). Weighting by inverse variance or by sample size in random-effects meta-analysis. Educational and Psychological Measurement, 70, 56-73. https:// doi.org/10.1177/0013164409344534

Micceri, T. (1989). The unicorn, the normal curve, and other improbable creatures. Psychological Bulletin, 105, 156-166. https://doi.org/10. 1037/0033-2909.105.1.156

Moè, A., \& Pazzaglia, F. (2006). Following the instructions!: Effects of gender beliefs in mental rotation. Learning and Individual Differences, 16, 369-377. https://doi.org/10.1016/j.lindif.2007.01. 002

R Core Team. (2018). R: A language and environment for statistical computing. Vienna, Austria: R Foundation for Statistical Computing. Retrieved from https://www.R-project.org/ 
Rosenthal, R., \& Rosnow, R. L. (1991). Essentials of behavioral research: Methods and data analysis (2nd ed.). New York, NY: McGraw Hill.

Rubio-Aparicio, M., Marín-Martínez, F., Sánchez-Meca, J., \& LópexLópez, J. A. (2018). A methodological review of meta-analyses of the effectiveness of clinical psychology treatments. Behavior Research Methods, 50, 2057-2073. https://doi.org/10.3758/ s13428-017-0973-8

Ruscio, J., \& Mullen, T. (2012). Confidence intervals for the probability of superiority effect size measure and the area under a receiver operating characteristic curve. Multivariate Behavioral Research, 47, 201-223. https://doi.org/10.1080/00273171.2012.658329

Sánchez-Meca, J., \& Marín-Martínez, F. (1998). Weighting by inverse variance or by sample size in meta-analysis: A simulation study. Educational and Psychological Measurement, 58, 211-220. https://doi.org/10.1177/0013164498058002005

Sánchez-Meca, J., Marín-Martínez, F., \& Chacón-Moscoso, S. (2003). Effect-size indices for dichotomized outcomes in meta-analysis. Psychological Methods, 8, 448-467. https://doi.org/10.1037/1082989X.8.4.448

Sawilowsky, S. S., \& Blair, R. C. (1992). A more realistic look at the robustness and Type II error properties of the $t$ test to departures from population normality. Psychological Bulletin, 111, 352-360. https://doi.org/10.1037/0033-2909.111.2.352
Staudte, R. G., \& Sheather, S. J. (1990). Robust estimation and testing. New York, NY: Wiley.

Tiku, M. (1964). Approximating the general non-normal variance-ratio sampling distributions. Biometrika, 51, 83-95. https://doi.org/10. 1093/bio-met/51.1-2.83

Van den Noortgate, W., \& Onghena, P. (2003). Multilevel meta-analysis: A comparison with traditional meta-analytical procedures. Educational and Psychological Measurement, 63, 765-790. https://doi.org/10.1177/0013164403251027

Veroniki, A. A., Jackson, D., Bender, R., Kuss, O., Langan, D., Higgins, J. P., Salanti, G. (2018). Methods to calculate uncertainty in the estimated overall effect size from a random-effects meta-analysis. Research Synthesis Methods, 10, 23-43

Viechtbauer, W. (2007). Hypothesis tests for population heterogeneity in meta-analysis. British Journal of Mathematical and Statistical Psychology, 60, 29-60. https://doi.org/10.1348/000711005X64042

Wilcox, R. R. (1987). New designs in analysis of variance. Annual Review of Psychology, 38, 29-60. https://doi.org/10.1146/annurev. ps.38.020187.000333

Publisher's note Springer Nature remains neutral with regard to jurisdictional claims in published maps and institutional affiliations. 